\title{
A 3500-year-old leaf from a Pharaonic tomb reveals that New Kingdom Egyptians were cultivating domesticated watermelon
}

3

4

Authors: Susanne S. Renner ${ }^{1}$, Oscar A. Pérez-Escobar ${ }^{2} *$, Martina V. Silber ${ }^{1}$, Mark Nesbitt ${ }^{2}$, Michaela Preick ${ }^{3}$, Michael Hofreiter ${ }^{3}$, Guillaume Chomicki ${ }^{4,5 *}$

\section{Affiliations:}

${ }^{1}$ Department of Biology, Institute for Systematic Botany and Mycology, University of Munich (LMU), Menzinger Str. 67, 80638 Munich, Germany. ${ }^{2}$ Royal Botanic Gardens, Kew, Richmond, TW9 3AE, UK. ${ }^{3}$ Faculty of Mathematics and Natural Sciences, Institute for Biochemistry and Biology, University of Potsdam, 14476 Potsdam, Germany. ${ }^{4}$ Department of Plant Sciences, University of Oxford, South Parks Road, Oxford OX1 3RB, UK; and ${ }^{5}$ The Queen's College, High Street, Oxford OX1 4AW, UK.

*These authors contributed equally to the study.

Correspondence: renner@1mu.de (SSR) or guillaume.chomicki@gmail.com (GC).

\begin{abstract}
Domestication of the watermelon (Citrullus lanatus) has alternatively been placed in South Africa, the Nile valley, or more recently West Africa, with the oldest archeological evidence coming from Libya and Egypt. The geographic origin and domestication of watermelons has therefore remained unclear. Using extensive nuclear and plastid genomic data from a 3,560year-old Citrullus leaf from a mummy's sarcophagus and skimmed genomes for representatives of the seven extant species of Citrullus, we show that modern cultivars and the ancient plant uniquely share mutations in a lycopene metabolism gene (LYCB) affecting pulp color and a stop codon in a transcription factor regulating bitter cucurbitacin compounds. This implies that the plant we sequenced had red-fleshed and sweet fruits and that New Kingdom Egyptians were cultivating domesticated watermelons. The genomic data also identify extant Sudanese watermelons with white, sweet pulp as the closest relatives of domesticated watermelons.

Archaeogenomics | plant domestication | ancient plant genome | Africa | New Kingdom Egypt | Pharaonic tomb

\section{Significance statement [120 words]}

With some 197.8 million tons in 2017, watermelon, Citrullus lanatus, is among the World's most important crops, yet its area of origin and domestication have remained unclear, with competing hypotheses favoring South Africa, West Africa, Central Africa, or the Nile valley. We generated extensive nuclear and plastid genomic data from a 3500-year-old leaf from a Pharaonic sarcophagus and performed genome skimming for representatives of all other Citrullus species to compare key genes involved in fruit bitterness and color. White-fleshed, non-bitter melons from southern Sudan are the closest relatives of domesticated watermelon, and the ancient genome shares unique alleles with a red-fleshed, non-bitter domesticated form (but no wild forms), implying that $18^{\text {th }}$ Dynasty Egyptians were cultivating domesticated watermelon by 3500 years ago.
\end{abstract}




\section{7 \\ Introduction}

Among the World's most important cucurbit fruit crops is the watermelon (Citrullus lanatus). With a production of 197.8 million tons in 2017, watermelon accounts for over a third of the global tropical fruit production (1). Its geographic origin and time of domestication, however, remain contentious (2-7, supplementary text). Competing hypotheses have favored South Africa (7, 8), Central Africa (2) or the Nile valley (9-15). More recently, molecular data have pinpointed West Africa $(16,17)$ as the region of the crop's origin, yet archaeological evidence is restricted to Egypt and Libya. Besides C. lanatus, the genus Citrullus contains six other species, of which four (C. amarus, C. ecirrhosus, C. naudinianus, and C. rehmii) occur mostly in the Namib-Kalahari region, one (C. mucosospermus) in West Africa (Benin, Ghana, and Nigeria), and one (C. colocynthis) from northern Africa to west India (Fig. 1A) and naturalized also in Australia $(6,17)$. All have white pulp that cannot be eaten raw due to the presence of terpene bitter compounds called cucurbitacins. Only fruits of $C$. тисоsospermus are sometimes not bitter, but instead bland-tasting (18). Citrullus lanatus itself is cultivated worldwide, but wild progenitors remained elusive despite the resequencing of 20 accessions from germplasm collections (16). Putative wild forms with small fruits and sweet white pulp have been reported since the $19^{\text {th }}$ century from the Kordofan region, a former province of Sudan, bordering North and South Darfur $(6,9-15)$ (Fig. 1A, B), but their genetic affinity to domesticated and other watermelons has not been tested. Moreover, no wild species of Citrullus with sweet pulp have been found in South Africa.

That the Egyptians ate domesticated watermelons is suggested by at least two wall paintings (Fig. 1C-D; Supplementary text) as well as Citrullus leaves from a tomb at Deir elBahari near Luxor (Theban Tomb number 320) first identified by the German archaeobotanist Georg August Schweinfurth in the $19^{\text {th }}$ century $(10,11,19-21)$ (Fig. 1B). The leaves were laying on the mummy of "Unknown Man C" in a re-used coffin inscribed for a priest Nebseni. On the evidence of embalming techniques and the coffin type the leaves may date to the early $18^{\text {th }}$ Dynasty (c. 3493-3470 BP; see Supplementary text). Finding leaves associated with mummies, however, is insufficient evidence that Egyptians consumed raw, sweet watermelons, since pharaonic tombs contain many kinds of plants that were not eaten as food, instead used as medicine or for other purposes (10). The closest known relative of the modern watermelon is a form from West Africa $(16,17)$ with white, usually bitter or bland pulp, and seeds that are used in West African 'egusi' stews (18).

To determine whether ancient Egyptians already consumed sweet, red watermelons and test whether sweet white-fleshed Sudanese Citrullus populations represent potential 
81 watermelon progenitors, we generated skimmed genome data $(\sim 14 \mathrm{~Gb})$ for all Citrullus

82 species, including the Unknown Man C mummy sample ( $\sim \mathrm{Gb})$ and accessions from North

83 and South Darfur (Supporting Information Table S1) hypothesized to be the wild progenitor

84 of watermelons $(6,9-15)$. The specific goals were to generate a strongly supported phylogeny,

85 probe the phylogenetic placements of the Unknown Man C mummy sample and Kordofan

86 melons, and searched bitterness and red-flesh markers in the $\sim 3500$-year-old sample.

87

\section{Results and discussion}

89 The 3500-year-old priest Unknown Man C mummy melon yielded good quality DNA with 90 relatively low rates of deamination (SI Appendix Figs. S1, S2). Because aDNA yields short 91 reads, we followed approaches used in other aDNA studies $(22,23)$ by mapping read data 92 against target sequences from the watermelon genome (16). The targets consisted of plastid 93 genes, a set of 143 nuclear genes for the phylogenomic analyses, and a set of 73 genes of 94 interest linked to fruit bitterness and color. We recovered a complete plastid genome for the 95 3500-year-old leaf (SI Appendix Fig. S3), and its nuclear sequences were sufficiently good so 96 that we could recover both our target genes for phylogenomics and our genes of interest

97 related to cucurbitacin and lycopene pathways. This enabled to probe the phylogenetic

98 position of the 3500-year-old Citrullus sample, and test whether its fruits were bitter or sweet, 99 and red or white-fleshed.

$100 \quad$ For the phylogenetic analyses of Citrullus, we assembled entire plastid genomes (121

101 genes and 33 spacers) and 143 single copy nuclear genes (SI Appendix, dataset 1) and

102 confirmed that nuclear genes are orthologs in reciprocal best alignment heuristic searches

103 (Materials and Methods, SI Appendix, dataset 2). Maximum Likelihood (ML) nuclear and

104 plastid phylogenies revealed the same topology (SI Appendix, Fig. 2A; Fig. S4), and a

105 backbone similar to an earlier phylogeny based on 11 genes that lacked any East African

106 samples (17). A coalescence-based tree also supported the same topology as ML analyses (SI

107 Appendix Fig. S5). These phylogenomic analyses revealed that the Unknown Man C mummy

108 sample is sister to modern domesticated watermelon (Fig. 2A; SI Appendix Figs. S4, S5) and

109 both together are sister to the Sudanese sweet and white-fleshed Kordofan melons (Fig. 2A;

110 C. lanatus subsp. cordophanus). All three form the sister clade to the West African $C$.

111 mucosospermus (Fig. 2A). These analyses revealed that the 3500-year-old Unknown Man C

112 mummy sample indeed represents a cultivated watermelon and that Kordofan melons $(C$.

113 lanatus subsp. cordophanus) are the closest known relative of the watermelon and likely 
114 descendants of a progenitor population of the domesticated watermelon. All Citrullus species 115 are illustrated in Fig. 2B-I.

116 To find direct evidence of the timing of watermelon domestication, we next

117 investigated whether the 3500-year-old leaf belonged to a watermelon with bitter vs. sweet

118 fruits and white $v s$. red flesh. Bitterness in cucurbits depends on terpene compounds called

119 cucurbitacins. The $B i$ gene, which encodes an oxidosqualene cyclase $(O S C)$ that catalyzes the

120 first committed step in cucurbitacin $\mathrm{C}$ biosynthesis is critical for determining bitterness (24-

121 26). Two bHLH transcription factors ( $B l$ and $B t)$ regulate Cucurbitacin $\mathrm{C}$ biosynthesis by

122 upregulating $B i$ expression in the leaves $(B l)$ and fruits $(B t)$ directly via binding to the E-box

123 elements of the $B i$ promoter $(25,26)$. In addition to upregulating $B i$, the watermelon $C l B l$ and

$124 \mathrm{ClBt}$ upregulate most other cucurbitacin metabolism genes, including the two cytochrome

$125 \mathrm{P} 450$ enzymes that convert cucurbidienol in cucurbitacin precursor $(\mathrm{Cl} 890 \mathrm{~A}$ and $\mathrm{Cl} 890 \mathrm{~B}$

126 genes in watermelon) and the acyltransferase that convert the cucurbitacin precursor in

127 cucurbitacin E (26). In cucumber (Cucumis sativus), honey melon (Cucumis melo), and

128 watermelon, examination of different lines with varying bitterness revealed that domestication

129 of non-bitter cucumber occurred via a nucleotide substitution leading to a premature stop

130 codon in the $B t$ gene resulting in a truncated, non-functional protein (24-26). A genomic

131 analysis comparing cucumber, honey melon, and watermelon (producing cucurbitacins C, B

132 and E, respectively), revealed a domestication sweep at the $B t$ locus, with the loss of

133 bitterness being due to convergent mutations at this locus (26).

134 We used a comparative-genomic approach to compare genes in the cucurbitacin

135 biosynthetic pathway and its regulation across Citrullus species and the ancient watermelon.

136 All cucurbitacin metabolic genes were conserved, with the same copy number across all

137 Citrullus species, including the 3500-year-old sample (Supplementary Dataset S1). To

138 probe whether the $\sim 3500$-year-old watermelon had bitter or sweet fruits, we compared its $B t$

139 gene to that of the other Citrullus species. Most Citrullus species have two copies of ClBt,

140 with the exception of $C$. rehmii and the clade comprising C. mucosospermus, the Kordofan

141 melon, and the modern and ancient watermelon, which have a single copy (Fig. 2J). The

142 second copy appears non-functional (Fig. 2J, Supplementary Dataset S1). The analyses

143 further revealed that the 3500-year-old mummy sample has a single nucleotide substitution

144 leading to a premature stop codon in the Bt gene (Fig. 2J, SI Appendix Fig. S6A and dataset

145 1), implying that it had non-bitter fruits. The Kordofan melons (subsp. cordophanus) also

146 have the premature stop codon, matching their consistently sweet pulp (13). The fruits of the

147 West African C. mисоsospermus are usually bitter, but over $20 \%$ have acid, plain, or sweet 
148 pulp (18: Table 5), matching our finding of a stop coding in its Bt gene. The particular plant

149 that we sequenced, E.G. Achigan-Dako 809AA603 (SI Appendix, Table S1), indeed had non-

150 bitter pulp (18; Achigan-Dako, pers. comm. SSR, 22 Nov. 2018). These results suggest a

151 scenario wherein loss of pulp bitterness was a pre-adaptation for the domestication of

152 watermelon, implying that early farmers probably took into cultivation non-bitter plants from

153 the wild. Thus, our comparative analyses of ClBt genes across all Citrullus species suggests

154 that the loss of bitterness resulted from initial selection of a non-bitter wild from, rather than

155 selection occurring during cultivation as may have been the case in cucumber $(25,26)$.

156 The red pulp color in modern watermelon is due to lycopene accumulation, likely by

157 blocking the conversion of lycopene into $\beta$-carotene, a step mediated by the enzyme LCYB

158 (lycopene $\beta$-cyclase) $(16,27,28)$. LCYB is encoded by the lcyb gene, and watermelon

159 accessions with red flesh are characterized by having a unique A $>$ C substitution in the $l c y b$

160 gene on chromosome 4, resulting in a Valine instead of a Phenylalanine at position 226 (27).

161 To determine whether the 3500-old watermelon was red-fleshed, we sought all lycopene

162 metabolic genes across our Citrullus genomes. This revealed a high conservation of these

163 metabolic genes, with the same copy number for all genes in the lycopene pathway across all

164 species (Supplementary Dataset S1). We found that the 3500-year-old mummy sample and

165 the modern watermelon share the key substitution in LCYB linked to lycopene accumulation

166 (Fig. 2K, SI Appendix Fig. S6B and SI dataset 1), while all other Citrullus species

167 including the Kordofan watermelons lack this mutation and have white to greenish pulp. This

168 reveals that New Kingdom Egyptians were cultivating a red, sweet watermelon 3500 years

169 ago.

\section{Conclusion}

172 Our study reveals that New Kingdom Egyptians cultivated a red, sweet watermelon at least

1733500 years ago. It also shows that likely progenitor populations still exist in the upper Nile

174 valley, in what is now the Darfur region of Sudan. The sweet, red watermelon may have been

175 domesticated there and the use of this plant then could have spread northward along the Nile

176 valley. Our finding that the 3500-year-old leaf belonged to a red-fleshed and sweet

177 watermelon is consistent with two Egyptian wall paintings dating back to $4350 \mathrm{BP}$ that have

178 been interpreted as showing watermelons (Fig. 1C-E; Supplementary text). They show

179 elongate fruits with the characteristic white stripes of watermelons and were served on trays,

180 suggesting they were eaten raw. Watermelon may have been domesticated even earlier, given

181 a watermelon seed found at Uan Muhuggiag in the Tadrart Acacus area in Libya that is 
182 between 5000 and 8650 years-old (29). Uan Muhuggiag lies $2240 \mathrm{~km}$ west of the $18^{\text {th }}$

183 Dynasty Theban necropolis of Deir el-Bahari where the genomic data here produced

184 originated. Recent work on barley (22) and maize (23), and the present study on the

185 watermelons, exemplifies the power of integrating collections-based phylogenomics with

186 archaeological data and comparative genomics to resolve the domestication history of modern

187 crops. The search for wild watermelon germplasm for engineering more disease-resistant

188 varieties now should concentrate on the Darfur region of Sudan.

189

190

Methods

191 Plant taxon sampling. Material sequenced for this study is listed in Supporting Information

192 Table S1, which also reports GenBank accession number for the skimmed genomes. Citrullus

193 taxonomy follows Chomicki \& Renner (17) and Renner et al. (6). We sampled all seven

194 Citrullus species using herbarium-verified samples all of which are linked to permanently

195 kept vouchers. This is essential, since very few -if any- germplasm accessions (for instance,

196 Citrullus accessions in the National Plant Germplasm System Germplasm Resources

197 Information Network [GRIN; http://www.ars-grin.gov/npgs]) are linked to herbarium voucher

198 specimens, leading to problems of unverifiability (see for instance Renner et al. (ref. 6)).

199

200

Ancient DNA extraction. To document the leaf fragment received from Kew's economic

201 plant collection, we first took high resolution photographs (Fig. 1B). A small piece of a leaflet

202 $\left(<1 \mathrm{~cm}^{2}, 5.8 \mathrm{mg}\right.$ ) was ground with a Retsch mill (Retsch MM 400). DNA extraction was performed following Wales et al. (31) (modified by Pedersen et al. ref. 32) and Dabney at al. (33). For the digestion treatment, a lysation buffer containing $0.5 \%(\mathrm{w} / \mathrm{v}) \mathrm{N}$-Lauroylsarcosine (Sigma Aldrich L9150-50G), 50 mM Tris-HCl (Thermo Fisher Scientific 15568025), $20 \mathrm{mM}$ EDTA (VWR E177-500MLDB) 150 mM NaCl (Thermo Fisher Scientific AM9760G), $3.3 \%$ 2-Mercaptoethanol (Sigma Aldrich 63689-25ML-F), 50 mM DL-Dithiothreitol (Sigma Aldrich D9779-250MG) and $0.25 \mathrm{mg} / \mathrm{mL}$ Proteinase K (Promega V3021) was applied to the leaflet powder as described in Wales et al. (31). DNA purification was performed according to Dabney at al. (33) with reduced centrifugation speed $(450 \mathrm{x} g$ ) as described in Basler et al.

\section{1 (34).}

Ancient DNA library preparation and sequencing. DNA extract was converted to an

214 Illumina sequencing library using the single-stranded approach described in Korlević et al.

215 (35). The protocol included the treatment with Uracil-DNA-Glycolase (New England Biolabs 216 M0279) to remove Uracil residues and Endonuclease VIII (New England Biolabs M0299) to 217 cleave DNA strands at abasic sites. $2.5 \mathrm{U} / \mu \mathrm{l}$ of Circligase II (Biozym 131406) was used for 218 the fill-in reaction and carried out overnight. A quantitative PCR was performed on a 219 PikoReal 96 Real-Time PCR machine (Thermo Fisher Scientific TCR0096) using $0.2 \%$ of 220 the unamplified library following this thermal profile: $10 \mathrm{~min}$ initial denaturation step at 95 $221{ }^{\circ} \mathrm{C}$, followed by 40 cycles of: $15 \mathrm{~s}$ at $95{ }^{\circ} \mathrm{C}, 30 \mathrm{~s}$ at $60{ }^{\circ} \mathrm{C}$, and $1 \mathrm{~min}$ at $72{ }^{\circ} \mathrm{C}$. The 222 quantitative PCR reaction mix contained a final volume of $10 \mu \mathrm{L}: 1 \mu \mathrm{L}$ of diluted library, $1 \mathrm{x}$ 223 SYBR Green qPCR Master Mix (Applied Biosystems 4309155), $0.5 \mu \mathrm{M}$ of each primer IS7 
and IS8. Three replicates of each library were used. Indexing PCR was performed by the appropriate number of cycles adding 8 bp indices to 5' and 3' adapters. PCR and final concentrations are the same as described (36), but with a final volume of $80 \mu \mathrm{L}$ using 20 $\mu \mathrm{L}$ of template. DNA sequencing was performed on an Illumina NextSeq 500 sequencing platform, using the 500/550 High Output v2 kit (75 cycles, Illumina FC-404-2005), with a custom read-1 (36) and a custom index-2 (37) sequencing primer. Extraction and library building was performed in the ancient DNA facility of the University in Potsdam; negative controls were included in all steps.

Genome skimming of Citrullus. We extracted genomic DNA from all Citrullus samples except the 3500-year-old sample using the Quiagen DNeasy Plant kit, following the manufacturer's protocol. Genome skimming was performed by the company Genewiz (New Jersay, USA) in Germany. Paired (2x150 bp) DNA libraries were sequenced on an Illumina HiSeq platform and $350 \mathrm{~GB}$ of sequence data were produced. An average of 100 million reads were produced for each sample.

Plastid data processing, assembly and annotation. The Illumina raw reads were quality filtered using Trim Galore v.0.4 (38), discarding sequences with averaged phred33 score below 20. Pre- and post-trimming read quality was assessed using FASTQC v.0.1 (39). Whole plastid genomes were assembled by blasting quality-filtered reads against a fully annotated reference plastid genome of $C$. lanatus (GenBank accession NC032008) using the tool blastn and a maximum number of target sequences of one. We obtained the best scoring blast hits from the blastn output by filtering all hits by bitscore and query coverage values of 100 and $80 \%$, respectively. Best scoring reads were mapped against the reference plastid genome using the Geneious mapping tool (40) and the following parameters: five iterations, minimum mapping quality of 30 , maximum gap size of 100 , and maximum mismatches per read of $40 \%$. Consensus genome sequences were assembled following a modified statistical base-calling approach of $\mathrm{Li}$ et al. (41), i.e. minimum depth coverage of 10, and bases matching at least $50 \%$ of the reference sequence. Plastid genomes were fully annotated by transferring intron, exon, and spacer annotation from the reference plastid genome to the denovo assembled plastids.

Plastid and nuclear phylogenomics of Citrullus. De-novo plastid genomes were aligned with Mauve using a progressive algorithm and assuming collinearity (42). The resulting $\sim 150,000$ bp alignment was subjected to Maximum Likelihood (ML) tree inference in RAxML v8.0 (43), using the GTR substitution model, 25 gamma categories, and 1,000 bootstrap replicates. Nuclear phylogenomic inferences relied on a selection of 143 out of 353 nuclear CDS known to be present in low copy numbers across angiosperms (44), all assembled from the trimmed read data using the python package Hybpiper (45). The 143 genes (SI dataset 1) were selected based on the proportions of missing data (i.e. alignments including more than five sequences with $50 \%$ of their length missing and less than five taxa were excluded), and phylogenetic informativeness (PI) profiles (i.e. gene alignments with disproportionate substitution rates were excluded) following Townsend (46). The PI profiles are provided in SI Appendix, Fig. S7. Hybpiper-achieved gene assembly by mapping the trimmed reads against the set of targeted CDSs using the Burrows-Wheeler alignment 

SPAdes (48), relying on default settings. Whenever multiple copies of the target genes are present in the genome, HybPiper raises paralog warnings during the contig assembly step. Here, no paralog warnings were issued for any of the 143 low copy nuclear genes. Because the Illumina sequencing of the ancient $C$. lanatus sample produced very short reads (max length of 75 bp; SI Appendix, Fig. S1), we carried out low-copy-gene assembly using the Geneious mapping algorithm, using customized settings. Gene mappings and assemblies were all checked by eye and carefully curated. To determine whether the selected set of low copy nuclear genes are in fact orthologs, we carry out orthology analyses in the software ProteinOrtho (49), which relies on a reciprocal best alignment heuristic approach. No paralogues were detected by ProteinOrtho (SI Appendix, dataset 2).

Phylogenomic analyses of the unlinked gene alignments matrix relied on ML and coalescence-based methods. We generated ML trees for every nuclear gene alignment as well as the concatenated nuclear matrix using the same settings as employed for plastid tree inference. Species tree analyses relied on the coalescence-based method ASTRAL III (50), using as input the annotated consensus gene trees derived from RAxML, with branches having Likelihood Bootstrap Support (LBS) < 10 collapsed. Gene tree conflict was visualized by plotting the local posterior probabilities derived from ASTRAL as pie diagrams at nodes. The final normalized quartet score is: 0.73 .

In-silico capture of genes relating to cucurbitacin biosynthesis and its regulation, lycopene synthesis and sugar metabolism and its regulation in Citrullus species

We followed the same read-data filtering and trimming procedure as used for the plastid data. Nucleotide deamination analysis and filtering of the ancient watermelon reads were carried out by the pipeline PALEOMIX (51) and using as a reference a genome assembly of a modern accession of $C$. lanatus (No. 97103, available at http://cucurbitgenomics.org/). The pipeline relied on the software Bowtie2 (52) for read

296 mapping and on mapDamage2 (53) for nucleotide deamination analysis. Genes involved in the cucurbitacin and lycopene synthesis were also captured in-silico following the same approach employed to data-mine low copy nuclear genes in the pipeline HybPiper. To attain this, we build a customized set of target genes obtained from Guo et al. (16) and Zhou et al. (26) (gene IDs are provided in SI Appendix, dataset 3). Gene copies in the ClBt gene were detected by HybPiper during the contig assembly step. No paralog warnings were produced for the remaining genes involved in the cucurbitacin and lycopene pathways (except for the ClBt gene for which some species had two copies-cf. Results and discussion).

\section{Acknowledgements}

306 We thank the egyptologists, R. Schiestl, University of Munich, Richard Parkinson, University of Oxford, and John H. Taylor, Department of Ancient Egypt and Sudan, The British

308 Museum, for consultation about the age of the mummy; and S. Bellot (Royal Botanic

309 Gardens, Kew) for advice on in-silico gene capture. This project was supported by the

310 German Science foundation (DFG grant RE 603/27-1) and the Elfriede and Franz Jakob

311 Foundation for research in systematic botany. G.C. is supported by a Glasstone Fellowship

312 and a Junior Research Fellowship at the University of Oxford. O.A.P.E. is supported by the

313 Lady Sainsbury Orchid Fellowship. 
Author Contribution

316 SSR and GC conceived the study with subsequent methodological contribution from OAPE;

317 SSR, MVS, MP gathered data; OAPE, GC, SSR analyzed data, SSR, GC, MH provided

318 funding; SSR and GC wrote the manuscript with edits from all authors.

\section{Additional information}

321

322

323

324

Supplementary Information accompanies this paper at https: xxx

Competing interests: The authors declare no competing financial interests.

\section{References:}

1. FAO, http://www.fao.org/faostat/en/\#data/QC, accessed 29 Jan. 2019

2. Hancock JF (2012) Plant evolution and the origin of crop species. 2nd edn. Wallingford, UK \& Cambridge, MA, USA: CABI.

3. Dane F, Liu J. (2007) Diversity and origin of cultivated and citron type watermelon (Citrullus lanatus). Genet Resources Crop Evol 54:1255-1265.

4. Paris HS (2015) Origin and emergence of the sweet dessert watermelon, Citrullus lanatus. Ann Bot 116:133-148.

5. Paris HS (2016) Overview of the origins and history of the five major cucurbit crops: issues for ancient DNA analysis or archaeological specimens. Veg Hist Archaeobot 25:405-414.

6. Renner SS, Sousa A, Chomicki G (2017) Chromosome numbers, Sudanese wild forms, and classification of the watermelon genus Citrullus, with 50 names allocated to seven biological species. Taxon 66:1393-1405.

7. Bailey LH (1930) Three discussions in Cucurbitaceae. Gentes Herb 2:175-186.

8. Dane F, Lang P (2004) Sequence variation at cpDNA regions of watermelon and related wild species: Implications for the evolution of Citrullus haplotypes. Amer J Bot 91:1922-1929.

9. Schweinfurth G (1873) Sur l'origine de quelques plantes cultivées en l'Égypte. Bull. de l'Inst. Egypt. 12:200-206.

10. Schweinfurth G (1883) La flore de l'ancienne Egypte. Rev Sci 32:72-77.

11. Schweinfurth G (1883) The flora of ancient Egypt. Nature 28:109-114.

12. Schweinfurth G (1884) Ueber Pflanzenreste aus altaegyptischen Gra $\square$ bern. Ber. Deutsch. Bot. Ges. 2:351-371.

13. Ter-Avanesyan DV (1966) Arbuz Kordofanskyi Citrullus lanatus Mansf. ssp. cordophanus Ter-Avan. Bot Zhurnal 51:423-426.

14. Fursa TB (1972) K sistematike roda Citrullus Schrad. [On the taxonomy of genus Citrullus] Schrad. Bot. Zhurn. (Moscow \& Leningrad) 57:31-41.

15. Fursa TB, Gavrilyuk IP (1990) Phylogenetic relations of the genus Citrullus Schrad. based on the immunochemical analysis of seed proteins. Sborn Nauchn Trudov Prikl Bot Genet Selekts 133:19-26. [In Russian with English summary]

16. Guo S. et al. (2013) The draft genome of watermelon (Citrullus lanatus) and resequencing of 20 diverse accessions. Nat Genet 45:51-58. 
17. Chomicki G, Renner SS (2015) Watermelon origin solved with molecular phylogenetics including Linnaean material: Another example of museomics. New Phytol 205:526-532.

18. Achigan-Dako EG, Avohou ES, Linsoussi C, Ahanchede A, Vodouhe RS, Blattner FR. (2015) Phenetic characterization of Citrullus spp. (Cucurbitaceae) and differentiation of egusi-type (C. mucosospermus). Genet Resour Crop Ev 62:11591179 .

19. Maspero G (1889) Les momies royales de Deir El-Bahari. Collection XIX.

20. Keimer L. (1924) Die Gartenpflanzen im alten Ägypten. Vol. I. Hamburg-Berlin: Hoffmann und Campe Verlag.

21. Germer R (1988) Katalog der Altägyptischen Pflanzenreste der Berliner Museen. Ägyptologische Abhandlungen 47. Wiesbaden: Otto Harrassowitz.

22. Mascher M et al. (2016) Genomic analysis of 6,000-year-old cultivated grain illuminates the domestication history of barley. Nat Genet 48:1089-1093.

23. Ramos-Madrigal J, Smith BD, Moreno-Mayar JV, Gopalakrishnan S, Ross-Ibarra J, Gilbert MTP, Wales N. (2016) Genome sequence of a 5,310-year-old maize cob provides insights into the early stages of maize domestication. Curr. Biol. 26, 31953201.

24. Qi J et al. (2013) A genomic variation map provides insights into the genetic basis of cucumber domestication and diversity. Nat Genet 45:1510-1515.

25. Shang Y et al. (2014) Biosynthesis, regulation, and domestication of bitterness in cucumber. Science 346:1084-1088.

26. Zhou Y et al. (2016) Convergence and divergence of bitterness biosynthesis and regulation in Cucurbitaceae. Nat Plants 2:16183.

27. Bang H, Kim S, Leskovar D, King S (2007) Development of a codominant CAPS marker for allelic selection between canary yellow and red watermelon based on SNP in lycopene $\beta$-cyclase (LCYB) gene. Mol Breed 20:63-72.

28. Grassi S et al. (2013) Comparative genomics reveals candidate carotenoid pathway regulators of ripening watermelon fruit. BMC Genomics 14:781.

29. Wasylikowa K, van der Veen M (2004) An archaeobotanical contribution to the history of watermelon, Citrullus lanatus (Thunb.) Mats. \& Nakai (syn. C. vulgaris Schrad.). Veget Hist Archaeobot 13:213-217.

30. Naville E (1912) Papyrus funéraires de la XXI dynastie. Vol. 1. Le papyrus hiéroglyphique de Kamara, le papyrus hiératique de Neskhonsou au Musée de Caire. Paris, Ernest Leroux.

31. Wales N, Andersen K, Cappellini E, Ávila-Arcos MC, Gilbert PT (2014) Optimization of DNA recovery and amplification from non-carbonized archaeobotanical remains. PloS ONE 9:e86827.

32. Pedersen JS, Valen E, Velazquez AMV, Parker BJ, Rasmussen M, Lindgreen S, Lilje B, Tobin DJ, Kelly TK, Vang S, Andersson R. (2014) Genome-wide nucleosome map and cytosine methylation levels of an ancient human genome. Genome Res. 24, 454466.

33. Dabney J et al. (2013) Complete mitochondrial genome sequence of a Middle Pleistocene cave bear reconstructed from ultrashort fragments. Proc Natl Acad Sci USA 110:15758-15763. 
34. Basler N, Xenikoudakis G, Westbury MV, Song L, Sheng G, Barlow A (2017) Reduction of the contaminant fraction of DNA obtained from an ancient giant panda bone. BMC Res Notes 10:754.

35. Korlević P et al. (2015) Reducing microbial and human contamination in DNA extractions from ancient bones and teeth. Biotechniques 59:87-93.

36. Gansauge M-T, Meyer M (2013) Single-stranded DNA library preparation for the sequencing of ancient or damage DNA. Nat Protoc 8:737-748.

37. Paijmans JLA, Baleka S, Henneberger K, Barlow A (2017) Sequencing singlestranded libraries on the Illumina NextSeq 500 platform. arXiv:1711.11004v1.

38. Krueger F Trim Galore v.0.4 (2015) A wrapper tool around Cutadapt and FastQC to consistently apply quality and adapter trimming to FastQ files.

39. Andrews S (2010) FastQC: a quality control tool for high throughput sequence data.

40. Kearse M et al. (2012) Geneious Basic: an integrated and extendable desktop software platform for the organization and analysis of sequence data. Bioinformatics 28:1647-1649.

41. Li H, Ruan J, Durbin R (2008) Mapping short DNA sequencing reads and calling variants using mapping quality scores. Genome Res 18:078212.

42. Darling AC, Mau B, Blattner FR, Perna NT (2004) Mauve: multiple alignment of conserved genomic sequence with rearrangements. Genome Res 14:1394-1403.

43. Stamatakis A (2014) RAxML version 8: a tool for phylogenetic analysis and postanalysis of large phylogenies. Bioinformatics 30:1312-1313.

44. Johnson M, L. Pokorny L, Dodsworth S, Botigue LR, Cowan RS, Devault A, Eiserhardt WL, Epitawalage N, Forest F, Kim JT, Leebens-Mack JH (2019) A universal probe set for targeted sequencing of 353 nuclear genes from any flowering plant designed using k-medoids clustering. Syst Biol in press; bioRxiv, p.361618.

45. Johnson MG, Gardner EM, Liu Y, Medina R, Goffinet B, Shaw AJ, Zerega NJ, Wickett NJ (2016) HybPiper: Extracting coding sequence and introns for phylogenetics from high-throughput sequencing reads using target enrichment. Appl Plant Sci 4:1600016.

46. Townsend JP (2007) Profiling phylogenetic informativeness. Syst Biol 56:222-231.

47. Li H, Durbin R (2009) Fast and accurate short read alignment with Burrows-Wheeler transform. Bioinformatics 25:1754-1760.

48. Bankevich A et al. (2012) SPAdes: a new genome assembly algorithm and its applications to single-cell sequencing. J. Comput. Biol. 19, 455-477 (2012).

49. Lechner M, Findeiß S, Steiner L, Marz M, Stadler PF, Prohaska SJ (2011) Proteinortho: detection of (co-) orthologs in large-scale analysis. BMC Bioinformatics 12:124.

50. Zhang C, Rabiee M, Sayyari E, Mirarab S (2018) ASTRAL-III: polynomial time species tree reconstruction from partially resolved gene trees. BMC Bioinformatics 19:153.

51. Schubert M et al. (2014) Characterization of ancient and modern genomes by SNP detection and phylogenomic and metagenomic analysis using PALEOMIX. Nat protoc 9:1056.

52. Langmead B, Salzberg SL (2012) Fast gapped-read alignment with Bowtie 2. Nat Methods 9:357. 
53. Jónsson H, Ginolhac A, Schubert M, Johnson PL, Orlando L (2013) mapDamage2. 0: fast approximate Bayesian estimates of ancient DNA damage parameters. Bioinformatics 29:1682-1684.

\section{Figure legends}

Fig. 1 Distribution of Citrullus species, archaeological samples and illustrations of watermelon in Ancient Egypt. (A), Map showing the distribution of Citrullus species in Africa (C. colocynthis extends to West India). (B), An asterisk marks the location of the tomb at Deir el-Bahari near Luxor, Egypt, in which a leaf fragment was found on "Unknown Man C" (Cairo museum mummy CG61067) in the $19^{\text {th }}$ century and deposited in the Economic Plant Collection of Kew (9-12). Red triangles and patch mark the Kordofan region and locations with wild watermelons in North and South Darfur. Top, sarcophagus of Amenhotpou from Deir el-Bahari, a mummy from the same site (19). Bottom, the preserved leaf and the sequenced fragment seen from below (inset, magnification 250x). (C), Papyrus de Kamara (30), illustrating a Citrullus fruit (inset), interpreted as a wild watermelon by Keimer (20). (D-E), Wall illustrations from Pharaonic tombs. (D), Tomb of Chnumhotep, Saqqara, ca. 4450 BC. (E), Unspecified tomb from Meir, drawing by L. Manniche. Photo and drawing courtesy of L. Manniche, June 2018.

\section{Fig. 2 Phylogenomics of Citrullus reveals the watermelon progenitor and confirm that} New Kingdom leaves belong to Citrullus lanatus. (A) Phylogeny of all Citrullus species based on 143 low copy nuclear genes, rooted on the relevant outgroups (17) (see also SI Appendix, Figs. S1, S2). Values on branches are bootstrap support from 1,000 replicates under the same ML model applied to nuclear (left) or plastid DNA sequences (right). (B-I), Illustrations of all Citrullus species. (B) Citrullus lanatus (watermelon). (C) C. lanatus subsp. cordophanus (Kordofan melon). (D) C. mucosospermus (egusi melon). (E), C. amarus (citron melon [wild], preserving melon [domesticated]). (F) C. ecirrhosus (tendril-less melon). (G), C. rehmii. (H) C. colocynthis (colocynth). (I) C. naudinianus. (J-K) Key changes in genetic pathways underlying bitterness and lycopene synthesis in Citrullus. (J) Cucurbitacin pathway in Citrullus highlighting key changes in copy number and functionality of the fruit-specific transcription factor $\mathrm{ClBt}$ that controls fruit bitterness. (K) Lycopene pathway in Citrullus, and phylogenetic distribution of a key substation in LYCB (lycopene $\beta$-cyclase) gene that is linked to lycopene accumulation and controls fruit color.

SI Appendix consists of SI text, Table S1, SI figures 1-7, and supplementary datasets 1, 2, and 3. All DNA sequence alignments generated for the phylogenomic analyses as well as for the genes of interests. Dryad accession number forthcoming. 
